\title{
THE CAUCHY PROBLEM OF THE ONE DIMENSIONAL SCHRÖDINGER EQUATION WITH NON-LOCAL POTENTIALS
}

\author{
I.E. KOUGIAS
}

Department of Mathematics

University of Patras, Patras, Greece

(Received July 16, 1992)

ABSTRACT. For a large class of operators $A$, not necessarily local, it is proved that the Cauchy problem of the Schrödinger equation:

$$
-\frac{d^{2} f(z)}{d z^{2}}+A f(z)=s^{2} f(z), \quad f(0)=0, \quad f^{\prime}(0)=1
$$

possesses a unique solution in the Hilbert $\left(H_{2}(\Delta)\right)$ and Banach $\left(H_{1}(\Delta)\right)$ spaces of analytic functions 'in the unit disc $\Delta=\{z:|z|<1\}$.

KEY WORDS AND PHRASES. Cauchy problem, Schrödinger equation, Hardy-Lebesque spaces.

1991 AMS SUBJECT CLASSIFICATION CODES. 34A20, 34A15, 47B37.

\section{INTRODUCTION.}

Let $C[0, \pi]$ be the Banach space of continuous functions on the interval $[0, \pi]$. The norm of an element $f(x)$ of that space is defined by

$$
\|f\|=\sup _{x \in[0, x]}|f(x)| .
$$

Assume that $A$ is a linear bounded operator on $[0, \pi]$ not necessarily local, i.e., $A$ need not be the multiplication operator by a continuous function $a(x)$. It may, for instance, be an integral operator on $C[0, \pi]$. It is known that the Schrödinger equation:

$$
-\frac{d^{2} f}{d x^{2}}+A f(x)=s^{2} f(x)
$$

possesses a unique solution in $C[0, \pi]$ satisfying the initial conditions:

$$
f(0)=0, \quad f^{\prime}(0)=1
$$

provided that $|s|>c_{o}\|A\|, c_{o}=\max _{o<\alpha<1} \frac{1-\cos \alpha \pi}{\alpha}[1]$.

Also it is known [1] that the solution is bounded for every $s$ in the region:

$$
G=\left\{s:|s| \geq \alpha c_{o}\|A\|, \quad \alpha>1\right\} .
$$

The purpose of this paper is to prove similar results for the initial valued problem [(1.1),(1.2)] in the Hardy spaces $H_{2}(\Delta)$ and $H_{1}(\Delta)$. These are the spaces of analytic functions: $f(z)=$ $\sum_{n=1}^{\infty} \alpha_{n} z^{n-1}$ in the unit disk $\Delta=\{z:|z|<1\}$, which satisfy respectively the conditions: 
$\sum_{n=1}^{\infty}\left|\alpha_{n}\right|^{2}<\infty$ and $\sum_{n=1}^{\infty}\left|\alpha_{n}\right|<\infty$ or equivalently the conditions:

$$
\sup _{o<r<1} \int_{0}^{2 \pi}\left|f\left(r e^{i \vartheta}\right)\right|^{2} d \vartheta<\infty \text { and } \sup _{0<r<1} \int_{0}^{2 \pi}\left|f\left(r e^{i \vartheta}\right)\right| d \vartheta<\infty \text {, }
$$

for $r e^{i \vartheta}=z$.

\section{REDUCTION OF THE SCHRÖDINGER EQUATION.}

$$
-\frac{d^{2} f(z)}{d z^{2}}+A f(z)=s^{2} f(z)
$$

in $H_{2}(\Delta)\left(H_{1}(\Delta)\right)$ to an abstract operator form. (We follow the method prescribed in [2] and [3]).

Let $H$ denote an abstract separable Hilbert space with an orthogonal basis $\left\{e_{n}\right\}_{1}^{\infty}$ and let $V$ be the unilateral shift operator on $H$, i.e.,

$$
V: V e_{n}=e_{n+1}, n=1,2, \cdots, V^{*}: V^{*} e_{n}=e_{n-1}, \quad n \neq 1, \quad V^{*} e_{1}=0
$$

is the adjoint operator of $V$.

Every function $f(z)=\sum_{n=1}^{\infty} \alpha_{n} z^{n-1}$ in $H_{2}(\Delta)$ can be represented as follows: $f(z)=\left(f_{z}, f\right)$, where $(\cdot, \cdot)$ means the scalar product in $H$ and $f_{z}=\sum_{n=1}^{\infty} z^{n-1} e_{n},|z|<1$ are the eigenelements of $V^{*}$.

The space $H_{1}$ is the Banach space which consists of those elements $f=\sum_{n=1}^{\infty} \bar{\alpha}_{n} e_{n}$, in $H$, (overbar means complex conjugate), that satisfy the condition $\sum_{n}^{\infty}\left|\left(f, e_{n}\right)\right|<\infty$. This space under the isomorphism $f(z)=\left(f_{z}, f\right)$ is isomorphic to $H_{1}(\Delta)$.

The norm in $H_{1}$ is denoted by: $\|f\|_{1}=\sum_{n=1}^{\infty}\left|\left(f, e_{n}\right)\right|$. To any open set or dense linear manifold $E$ in $H\left(H_{1}\right)$ corresponds an open set or dense linear manifold $\bar{E}$ in $H_{2}(\Delta)\left(H_{1}(\Delta)\right)$. Suppose that $A$ is a mapping in $H_{2}(\Delta)\left(H_{1}(\Delta)\right)$ and $\bar{A}$ is a mapping in $H\left(H_{1}\right)$. Then if the relation $A f(z)=\left(f_{z}, \bar{A} f\right)$ holds $\forall f \in E$, we call $\bar{A}$ the abstract form of $A$. For example if $A$ is the differential operator $\frac{d^{2}}{d z^{2}}$ in $H_{2}(\Delta)$, i.e., $A f(z)=\frac{d^{2} f(z)}{d z^{2}}$, then $\bar{A}=\left(C_{o} V^{*}\right)^{2}=C_{o}\left(C_{o}+I\right) V^{* 2}$, where $C_{o}$ is the diagonal operator $C_{o} e_{n}=n e_{n}, n=1,2, \ldots$ (see for details in [2] and [3]).

Every bounded operator on $H_{2}(\Delta)(H)$ is defined on $H_{1}(\Delta)\left(H_{1}\right)$ and maps, in general, elements of $H_{1}(\Delta)\left(H_{1}\right)$ into $H_{2}(\Delta)(H)$.

The following properties follow easily:

(i) $H_{1}$ is invariant under the operators $V, V^{*}$ and $\|V\|_{1}=\left\|V^{*}\right\|_{1}=1$, where $\|A\|_{1}$ means the norm of an operator on $H_{1}$.

(ii) $H_{1}$ is invariant under every bounded diagonal operator $D e_{n}=d_{n} e_{n}, n=1,2, \cdots$ on $H$ and $\|D\|_{1}=\|D\|=\sup _{n}\left|d_{n}\right|$.

(iii) For every element $f(z)=\sum_{n}^{\infty} \alpha_{n} z^{n-1}$ in $H_{1}(\Delta)$ the uniform limit of the sequence $\sum_{i=1}^{n} \bar{\alpha}_{i} V^{i-1}$, i.e., $\lim _{n \rightarrow \infty} \sum_{i=1}^{n} \bar{\alpha}_{i} V^{i-1}$ exists and defines a bounded operator $f^{*}(V)=\bar{\alpha}_{1}+\bar{\alpha}_{2} V+\bar{\alpha}_{3} V^{2}$ $+\cdots$ on $H_{1}$. Moreover $\left\|f^{*}(V)\right\|_{1}=\|f\|_{1}$.

(iv) The null space of $V^{* k}$ in $H$ belongs to $H_{1}$.

Now we write equation (2.1) in the form:

$$
\frac{d^{2} f}{d z^{2}}+s^{2} f(z)-A f(z)=0 .
$$

The abstract form of equation (2.2) is the following:

or

$$
\left(\left(C_{o} V^{*}\right)^{2}-\bar{A}_{1}\right) f=0
$$

$$
\left(V^{* 2}-B_{1} \bar{A}_{1}\right) f=0 \text {, }
$$


where $\bar{A}_{1}=\bar{A}-s^{2} I$ and $B_{1}$ is the diagonal operator on $H: B_{1} e_{n}=\frac{1}{n(n+1)} e_{n}, n=1,2, \cdots$

This means that equation $(2.2)$ has a solution in $H_{2}(\Delta)\left(H_{1}(\Delta)\right)$ satisfying the conditions $f(0)=0, f^{\prime}(0)=1$ iff equation (2.4) has a solution in $H\left(H_{1}\right)$ satisfying the conditions:

$$
\left(f, e_{1}\right)=0, \quad\left(f, e_{2}\right)=1 .
$$

Note that $H_{1}$ is imbedded in $H$ in the sense that $f$ in $H_{1}$ implies $f$ in $H$ and $\|f\| \leq\|f\|_{1}$.

3. SOLUTION OF THE CAUCHY PROBLEM [(2.4), (2.5)] IN H AND $\mathrm{H}_{1}$.

THEOREM 1. The equation $\left(V^{*}-B_{1} \bar{T}_{1}\right) f=0$ has at least one solution in $I I$ which satistere the condition $\left(f, e_{1}\right)=0$.

PROOF. Set $f=V g$, then $\left(V_{g, f_{1}}\right)=\left(y \cdot V^{*} e_{1}\right)=(g, 0)=0$. Also $\left(V^{* 2}-B_{1} \bar{A}_{1}\right)\left(V^{\prime} !\right)=0$ mplies $V^{*}\left(I-V B_{1} \bar{A}_{1} V\right) g=0$.

Thus $\left(I-V B_{1} \bar{A}_{1} V\right) g=c e_{1}$.

Now since $B_{1}$ is compact, $V$ and $\bar{A}$ bounded the operator $V B_{1} \bar{A}_{1} V$ is compact and the Fredholm alternative implies that either: $\left(I-V B_{1} \bar{A}_{1} V\right) g=0$ for $g \neq 0$ or $\left(I-V B_{1} \bar{A}_{1} V\right)^{-1}$ exists and it is bounded.

In the first case $g \neq 0$ is a solution of equation (2.4). In the second case we have $g=c\left(I-V B_{1} \bar{A}_{1} V\right)^{-1} e_{1} \neq 0$ for $c \neq 0$.

Theorem 1 implies that the Schrödinger equation (2.1) has at least one solution in $H_{2}(\Delta)$ which satisfies the condition $f(0)=0$, for every real or complex $s$, and every bounded linear operator $\mathrm{A}$ on $\mathrm{H}_{2}(\Delta)$.

THEOREM 2. If $\left\|\bar{A}_{1}\right\|<2$, then equation (2.4) has a unique solution in $H$ which satisfies the conditions (2.5).

PROOF. Set $f=e_{2}+V^{2} g$, then obviously $\left(f, e_{1}\right)=0$ and $\left(f, e_{2}\right)=1$. Also from equation (2.4) we get: $-B_{1} \bar{A}_{1} e_{2}+I g-B_{1} \bar{A}_{1} V^{2} g=0$ which implies that

$$
\left(I-B_{1} \bar{A}_{1} V^{2}\right) g=B_{1} \bar{A}_{1} e_{2} .
$$

(i) If $\bar{A}_{1} e_{2}=0$, then $e_{2}$ is the unique solution in $H$ which satisfies the initial conditions, since $\left(I-B_{1} \bar{A}_{1} V^{2}\right) g=0$ implies that $g=0$.

(ii) If $\bar{A}_{1} e_{2} \neq 0$, then from equation (3.1) since $\left\|B_{1}\right\|=\frac{1}{2}$ and $\|\bar{A}\|<2$, we easily get that $\left\|B_{1} \bar{A}_{1} V^{2}\right\|<1$. Hence the inverse of $\left(I-B_{1} \bar{A}_{1} V^{2}\right)$ exists and it is bounded on $H$. Therefore $g=\left(I-B_{1} \bar{A}_{1} V^{2}\right)^{-1} B_{1} \bar{A}_{1} e_{2}, g \neq 0$ and $g$ is uniquely defined.

There has been defined, in [3], a class of bounded operators on $H\left(H_{1}\right)$ which have the socalled " $k$-invariant property." - Abstract forms of local potentials of the form: $A f(z)=a(z) f(z)$ are included in this class.

The importance of such operators is due to the fact that if $\bar{A}_{1}$ is $k$-invariant on the space $H_{2}$, then the operator $A_{2}=I-V^{2} B_{1} \bar{A}_{1}$ leaves invariant the space $H_{1}$ and when restricted on it, has a bounded inverse (see [3], Theorem 3.2).

DEFINITION. A bounded operator $\bar{A}$ on $H$ is called $k$-invariant iff its adjoint $\bar{A}^{*}$ has the property: $\bar{A}^{*} e_{i} \in M_{i+k-1}$, where $M_{i+k-1}$ is the subspace spanned by $\left\{e_{1}, e_{2}, \cdots e_{i+k-1}\right\}$, $i=1,2, \cdots$.

Such operators are the diagonal operators in the basis $\left\{e_{n}\right\}_{1}^{\infty}$, analytic functions of the shift $V$, algebraic combinations of the above and polynomial functions of $V^{*}$ of degree less than $k$.

In accordance with the above definition a bounded operator $A$ on $H_{2}(\Delta)\left(H_{1}(\Delta)\right)$ ) is called 2invariant iff its adjoint $A^{*}$ has the property: $A^{*} z^{i} \in\left\{1, z, z^{2}, \cdots, z^{i}\right\}$, where $\left\{1, z, z^{2}, \cdots, z^{i}\right\}$, is the subspace of $H_{2}(\Delta)\left(H_{1}(\Delta)\right)$ spanned by the elements $1, z, z^{2}, \cdots, z^{i}$. 
For example the operator:

$$
A=A f(z)=a f(z)+z f(z)+\frac{1}{z}(f(z)-f(0))
$$

is a 2-invariant self adjoint operator on $H_{2}(\Delta)$.

THEOREM 3. The Cauchy problem:

$$
\begin{gathered}
-\frac{d^{2} f(z)}{d z^{2}}+A f(z)=s^{2} f(z) \\
f(0)=0, \quad f^{\prime}(0)=1,
\end{gathered}
$$

where $A$ is any 2-invariant operator on $H_{1}(\Delta)$, has a unique solution in $H_{1}(\Delta)$ for every $s \in \mathrm{C}$.

This solution is bounded for every $z$ in the unit disc.

PROOF. The abstract form of (3.2) is:

$$
\left(V^{* 2}-B_{1} \bar{A}+s^{2} B_{1}\right) f=0
$$

and the conditions (3.3) are equivalent to

$$
\left(f, e_{1}\right)=0, \quad\left(f, e_{2}\right)=1 .
$$

Setting $f=e_{2}+V^{2} g$ which obviously satisfies the initial conditions (3.5) we get:

$$
\left(I-B_{1}\left(\bar{A}-s^{2}\right) V^{2}\right) g=B_{1}\left(\bar{A}-s^{2}\right) e_{2} .
$$

The operators $V, V^{*}$ and $B_{1}$ leave the space $H_{1}$ invariant. The same holds for the operator $\left(I-B_{1}\left(\bar{A}-s^{2}\right) V^{2}\right)$, which restricted on $H_{1}$ has a bounded inverse (see [3], Theorem 3.2). Also $B_{1}\left(\bar{A}-s^{2}\right) e_{2}=h \in H_{1}$ and the unique solution of (3.6) is given by: $g=\left(I-B_{1}\left(\bar{A}-s^{2}\right) V^{2}\right)^{-1} h$.

For every $f(z)=\sum_{n=1}^{\infty} \alpha_{n} z^{n-1} \in H_{1}(\Delta)$ we have: $|f(z)| \leq \sum_{n=1}^{\infty}\left|\alpha_{n}\right|=\|f(z)\|_{H_{1}(\Delta)}<\infty,|z| \leq 1$. This shows that the solution predicted by the theorem is bounded for $|z| \leq 1$.

ACKNOWLEDGEMENT. I wish to express my deep appreciation to Professor E.K. Ifantis, for his great help and supervision on the completion of this work.

\section{REFERENCES}

1. NASR, A.H., A study of one-dimensional Schrödinger equation with nonlocal potential, Siam J. Math. Anal. 15 (3) (1984), 459-467.

2. IFANTIS, E.K., An existence theory for functional differential equations and functional differential systems, J. Diff. Eq. 29 (1978), 86-104.

3. IFANTIS, E.K., Analytic solutions for nonlinear differential equations, J. Math. Anal. Appl. 124 (1987), 339-380. 


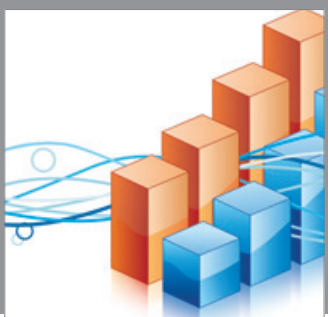

Advances in

Operations Research

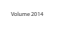

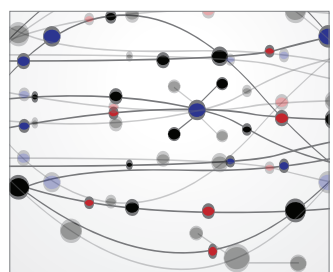

\section{The Scientific} World Journal
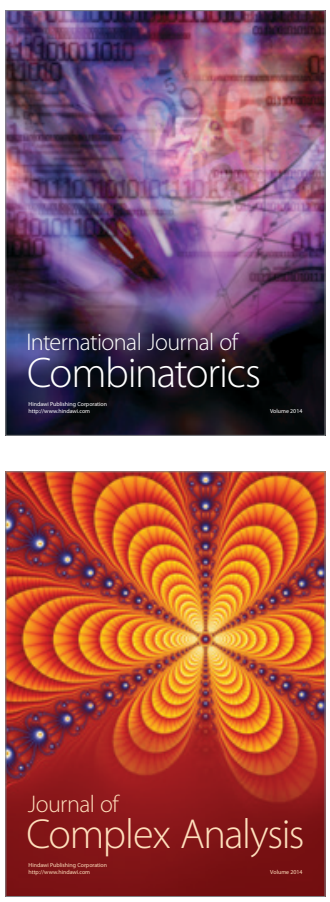

International Journal of

Mathematics and

Mathematical

Sciences
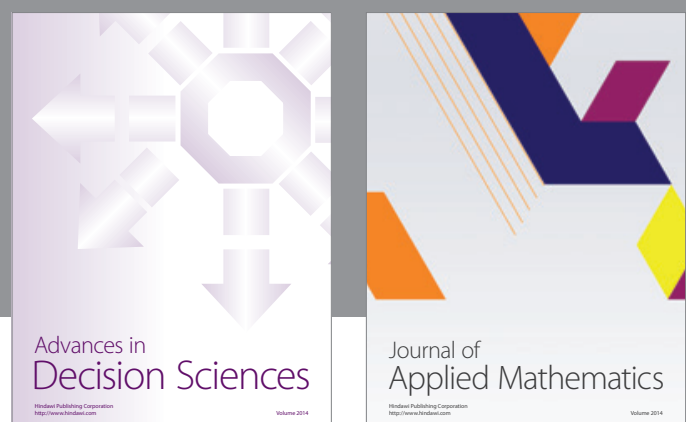

Journal of

Applied Mathematics
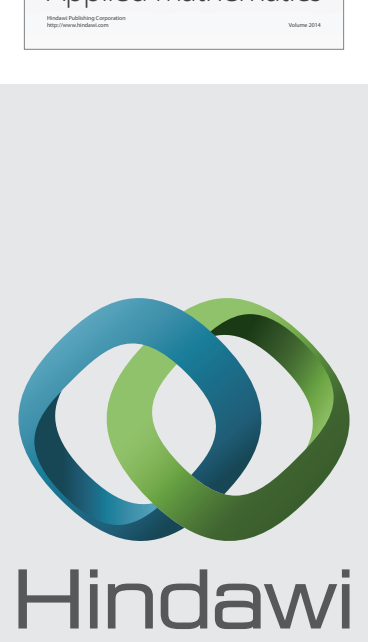

Submit your manuscripts at http://www.hindawi.com
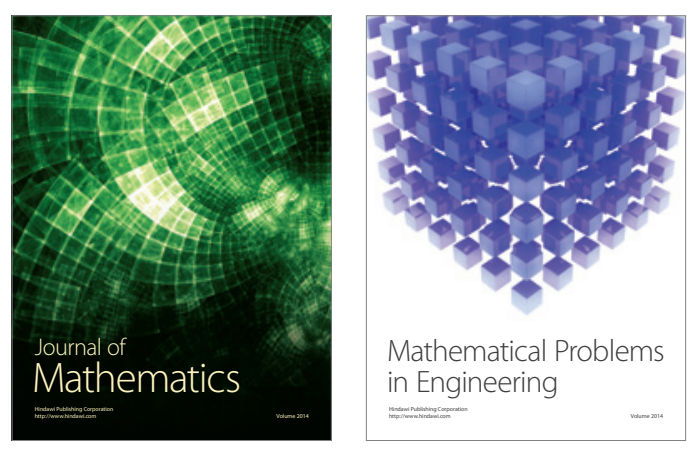

Mathematical Problems in Engineering
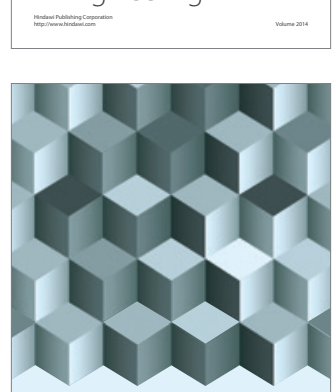

Journal of

Function Spaces
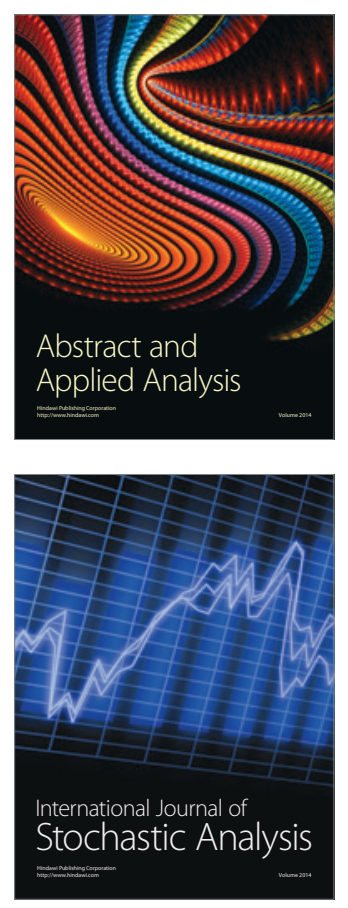

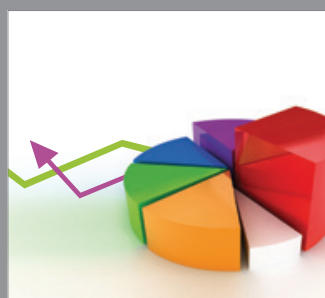

ournal of

Probability and Statistics

Promensencen
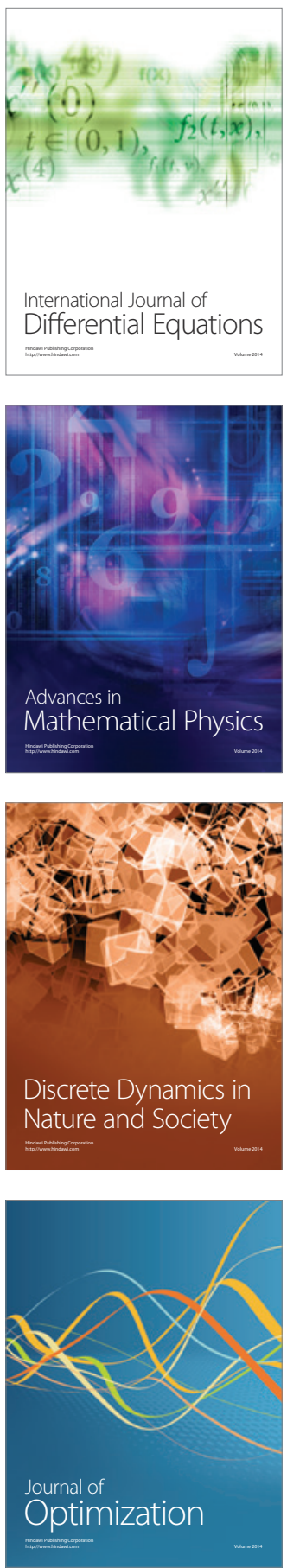